\title{
Subject-specific knee joint geometry improves predictions of medial tibiofemoral contact forces
}

\author{
Pauline Gerus ${ }^{1}$, Massimo Sartori², Thor F. Besier ${ }^{3}$, Benjamin J. Fregly ${ }^{4,5,6}$, Scott L. Delp 7 , \\ Scott A. Banks ${ }^{4,5,6}$, Marcus G. Pandy ${ }^{8}$, Darryl D. D'Lima ${ }^{9}$, and David G. Lloyd ${ }^{1}$ \\ ${ }^{1}$ Centre for Musculoskeletal Research, Griffith Health Institute, Griffith University, Southport, QLD, \\ Australia ${ }^{2}$ Bernstein Center for Computational Neuroscience, Georg-August University, \\ Göttingen, Germany ${ }^{3}$ Auckland Bioengineering Institute, University of Auckland, Auckland, New \\ Zealand ${ }^{4}$ Dept. of Mechanical \& Aerospace Engineering, University of Florida, Gainesville, FL, \\ USA ${ }^{5}$ Dept. of Biomedical Engineering, University of Florida, Gainesville, FL, USA ${ }^{6}$ Dept. of \\ Orthopaedics \& Rehabilitation, University of Florida, Gainesville, FL, USA ${ }^{7}$ Dept. of Mechanical \\ Engineering, Stanford University, Stanford, CA, USA ${ }^{8}$ Dept. of Mechanical Engineering, \\ University of Melbourne, Melbourne, VIC, Australia ${ }^{9}$ Shiley Center for Orthopaedic Research \& \\ Education at Scripps Clinic, La Jolla, CA, USA
}

\section{Abstract}

Estimating tibiofemoral joint contact forces is important for understanding the initiation and progression of knee osteoarthritis. However, tibiofemoral contact force predictions are influenced by many factors including muscle forces and anatomical representations of the knee joint. This study aimed to investigate the influence of subject-specific geometry and knee joint kinematics on the prediction of tibiofemoral contact forces using a calibrated EMG-driven neuromusculoskeletal model of the knee. One participant fitted with an instrumented total knee replacement walked at a self-selected speed while medial and lateral tibiofemoral contact forces, ground reaction forces, whole-body kinematics, and lower-limb muscle activity were simultaneously measured. The combination of generic and subject-specific knee joint geometry and kinematics resulted in four different OpenSim models used to estimate muscle-tendon lengths and moment arms. The subjectspecific geometric model was created from CT scans and the subject-specific knee joint kinematics representing the translation of the tibia relative to the femur was obtained from fluoroscopy. The EMG-driven model was calibrated using one walking trial, but with three different cost functions that tracked the knee flexion/extension moments with and without constraint over the estimated joint contact forces. The calibrated models then predicted the medial and lateral tibiofemoral contact forces for five other different walking trials. The use of subject-specific models with minimization of the peak tibiofemoral contact forces improved the accuracy of medial contact forces by $47 \%$ and lateral contact forces by $7 \%$, respectively compared with the use of generic musculoskeletal model.

(C) 2013 Elsevier Ltd. All rights reserved.

Corresponding author for Journal of Biomechanics: Pauline Gerus, PhD, Centre for Musculoskeletal Research, Griffith Health Institute, Griffith University, Southport Campus, QLD, Australia, 4222, Phone: +61 75552 7066, Fax: +61 75552 8674, p.gerus@griffith.edu.au.

Conflicts of interest statement

We wish to confirm that there are no known conflicts of interest associated with this publication and there has been no significant financial support for this work that could have influenced its outcome.

Publisher's Disclaimer: This is a PDF file of an unedited manuscript that has been accepted for publication. As a service to our customers we are providing this early version of the manuscript. The manuscript will undergo copyediting, typesetting, and review of the resulting proof before it is published in its final citable form. Please note that during the production process errors may be discovered which could affect the content, and all legal disclaimers that apply to the journal pertain. 


\section{Keywords}

EMG-driven modelling; knee joint model; contact force; muscle force

\section{Introduction}

Large joint contact forces are thought to be an important factor in the development and progression of osteoarthritis (Guilak, 2011; Hurwitz et al., 2001; Roemhildt et al., 2012; Solomon, 1976). The external knee adduction moment (KAM) has been used as a convenient surrogate for the medial-lateral load distribution at the knee and has been linked to the onset, progression, and severity of medial tibiofemoral osteoarthritis (Foroughi et al., 2009; Schipplein and Andriacchi, 1991). The KAM, estimated by inverse dynamics, does not account for the knee's other degrees of freedoms and the muscles' direct contribution to the knee contact forces, and does not always correlate strongly with medial contact force at the knee (Meyer et al., 2012). In this study, we hypothesised that computational neuromusculoskeletal models that include knee loading about multiple degrees of freedom and muscle forces may provide more accurate estimates of knee contact loads.

However, developing and validating these models is challenging because of the neuromusculoskeletal system complexity and inter-subject variability (Delp et al., 2007). The accuracy of computational models to predict tibiofemoral joint contact forces can be assessed using direct measures from instrumented total knee replacements (Fregly et al., 2012). Computational models that use generic anatomy tend to overestimate medial knee contact forces when compared to in vivo measurements (Fregly et al., 2012). Altered estimates of the muscle-tendon moment arms and muscle-tendon lengths from variations of musculoskeletal geometries have been reported for the knee (Pal et al., 2007) and hip joints (Duda et al., 1996; Scheys et al., 2011). Tsai et al. (2012) found that the use of moment arms estimated from magnetic resonance imaging provides a more accurate prediction of the net joint moment compared to the measured net joint moment. In this context, it is possible that the aforementioned contact force overestimations are due to an underestimation of muscle moment arms, resulting in higher muscle forces to generate the same net joint moment. In addition, joint kinematics estimation errors may affect load computations.

Computational models to estimate muscle forces can be broadly classified as; i) optimization method, which estimate a set of muscle activations based on an objective function (e.g. minimise muscle stress) (Crowninshield and Brand, 1981), or ii) electromyography (EMG) EMG-driven approach, which determines muscle activations based on recorded EMG signals (Lloyd and Besier 2003; Buchanan et al., 2004). In the case of musculoskeletal disorders, such as osteoarthritis, muscle activation strategies are highly variable and significantly different from normal healthy people (Zeni et al., 2010; Heiden et al., 2009). In this case, an EMG-driven approach appears warranted to account for an individual's unique muscle activation pattern (Kumar et al., 2012). The mapping from EMG to muscle force is not trivial and current EMG-driven methods use a calibration process to adjust EMG-toactivation and muscle-tendon parameters (Lloyd and Besier 2003). Parameter calibration attempts to match experimental joint moments of the ankle, knee and/or hip measured from inverse dynamics. However, this calibration is a limitation of EMG-driven modeling because the solution space is large and the matching of the knee flexion/extension joint moment does not necessarily ensure accurate joint contact force estimations. Indeed, even though EMG-driven approaches were found to predict joint moments very well, they nevertheless overestimated the medial tibiofemoral knee joint contact forces (Fregly et al, 2012). The influence of adding further constraints beyond the magnitude of the contact forces during the calibration process has not been investigated. 
The aim of this study was to investigate the influence of knee joint geometry, knee joint kinematics and calibration cost functions on the estimation of tibiofemoral contact forces using an EMG-driven neuromusculoskeletal approach. It was hypothesized that, subjectspecific knee joint geometry and/or knee joint kinematics would improve the accuracy of medial and lateral contact force predictions, compared to a generic model. We also hypothesized that a calibration cost function including a minimization of the peaks of medial and lateral contact forces would improve joint contact forces predictions.

\section{Method}

\subsection{Gait experiments}

This study used data previously collected from an adult male fitted with an instrumented total knee replacement (right knee, age 83, mass $68 \mathrm{~kg}$, height $1.7 \mathrm{~m}$ ) (Fregly et al., 2012). Institutional review board approval and the participant's informed, written consent were obtained prior to data collection.

We used data recorded from two gait tasks. The first was walking on an instrumented treadmill (Bertec, Columbus, USA) where a C-arm fluoroscope (GE Medical Systems, Salt Lake City, USA) was used to record rotations and translations of the tibia relative to the femur. The second task involved walking overground at a naturally selected walking speed ( $\mathrm{n}=$ six trials). The whole body segmental motion was recorded at $120 \mathrm{~Hz}$ using a VICON motion analysis system (Vicon, Oxford, UK). Ground reaction forces (GRF) were recorded at $1200 \mathrm{~Hz}$ from three force plates (Bertec, Columbus, USA), and surface EMG recorded at $1200 \mathrm{~Hz}$ using a 16-channel Bagnoli system (Delsys, Boston, USA) with custom double differential preamplified electrode leads. The motion capture markers were attached according to a full-body marker set reported by Besier et al. (2003) and EMG activity on the involved side was recorded from 8 muscles: biceps femoris long-head (BicFemlh), gastrocnemius lateralis (GasLat), gastrocnemius medialis (GasMed), rectus femoris (RectFem), semi-membranous (SemiMem), tensor fascia lata (TFL), vastus lateralis (VastLat), and vastus medialis (VastMed). Medial and lateral tibiofemoral contact forces were recorded at $120 \mathrm{~Hz}$, synchronously with motion capture, GRFs, and EMG.

\subsection{Description of the OpenSim models}

Various models were created with generic and subject-specific elements.

i. The generic geometry anatomical model was based on a full-body OpenSim model, which consisted of 14 rigid-linked skeletal segments with 37 degrees of freedom (DOF) (Hamner et al., 2010; Donnelly et al, 2012). This model was scaled in threedimensions to match each subject's anthropometry based on marker trajectories measured from motion capture and calculated hip, knee and ankle joint centres. The positions of the lower limb joint centres and axes were estimated from functional tasks (Besier et al., 2003; Donnelly et al, 2012). Of importance, the lower limbs had a 3 DOF ball joint for the hip and 1 DOF hinge joint for the ankle (Hamner et al., 2010; Donnelly et al, 2012). The knee joint kinematics are described in more detail below (see section 2.2 iii and $2.2 \mathrm{iv}$ ).

ii. The subject-specific geometry anatomical model was an adaptation of the generic full-body model. The upper body was the generic scaled model. However, the lower limb model was created using a subject-specific knee from the implant's geometry, bone geometry from CT scans (i.e., femur, tibia, fibula, and patella), and generic bone geometry for the other bodies. The position of the knee joint centre was located at the midpoint of the femoral condyles when the knee was in the fully extended posture, and the hip-joint centre was located in the centre of the femoral 
head (Arnold et al., 2010). The ankle-joint centre was calculated as for the scaled generic anatomical model. The vertical length of the femur, tibia, and fibula were adjusted to match the position of the calculated hip, knee, and ankle joint centres. The scale factor was 1.05 and 1.03 for the femur and tibia-fibula, respectively. Each muscle-tendon path was adjusted manually to fit with the new bone geometry using the bony landmarks from the generic model as a reference. The moving path definition of some muscles was adjusted to avoid penetration into bone. The translations of the patella as a function of knee flexion were redefined to fit the shape of the implant. The moving path of the quadriceps muscle group was modified to follow the new motion of the patella and to avoid penetration into the femur.

iii. The generic knee joint kinematic model had 3 rotational and 2 translational DOFs (Donnelly et al, 2012). The knee comprised of a sagittal planar joint with a flexion/ extension axis going through the knee joint centre and perpendicular to the plane. A spline defined the anterior-posterior and superior-inferior translations of the tibia in this plane as a function of knee flexion angle (Figure 1), which was the translation of the knee joint centre relative to the origin of the femur (femoral head) (Delp et al., 1990). The knees also had an internal/external rotation hinge joint with its axis going through the ankle joint and knee joint centres, and two hinge joints for adduction/abduction, the axes perpendicular to the tibial frontal plane with one going through the medial condyle contact point and the other through the lateral condyle contact point. The position of the medial and lateral condyle contact points were the same as used for the subject-specific knee (see below).

iv. for the subject-specific knee kinematic model the generic spline functions were adjusted to represent the experimental translations recorded using fluoroscopy without penetration between the femur and tibia (Figure 1). The position of the knee joint centre was not modified. Additionally, as in the generic knee, the medial and lateral condyle contact points were based on the inter-condyle distance and contact positions relative to the knee joint extracted from instrumented knee data and were $40 \mathrm{~mm}$ and $20 \mathrm{~mm}$ respectively (Zhao et al., 2007).

The combination of generic and subject-specific knee joint geometry and kinematics resulted in four different OpenSim models:

1. generic geometry and generic kinematics (G-Geom \& G-Kin)

2. generic geometry and subject-specific kinematics (G-Geom \& SS-Kin)

3. subject-specific geometry and generic kinematics (SS-Geom \& G-Kin)

4. subject-specific geometry and subject-specific kinematics (SS-Geom \& SS-Kin)

\subsection{Estimation of joint angles and joint moments in gait}

The OpenSim inverse kinematics and inverse dynamics analysis tools were used to estimate the joint kinematics and moments from the gait data (Delp et al., 2007). In the inverse kinematics solution all DOFs were free to move except at the knee where internal/external rotation and adduction/abduction were fixed and only flexion/extension permitted. However, this configuration enabled flexion/extension, internal/external rotation and adduction/ abduction moments at the medial and lateral condyle contact points to be determined via inverse dynamics. 


\subsection{Estimation of muscle-tendon forces}

The muscle force distribution problem (i.e. estimation of muscle forces) was solved using an EMG-driven approach, which has been described in details elsewhere (Lloyd and Besier, 2003; Buchanan et al, 2004, Winby et al, 2009; Kumar et al, 2012). Briefly, the model has four parts: (1) an anatomical model to estimate muscle-tendon lengths and moment arms, (2) an EMG-to-activation model to represent muscle activation dynamics, and (3) a Hill-type muscle model to characterize muscle-tendon contraction dynamics and estimate the forces in the muscle-tendon complex

As described above, OpenSim (SimTK, Stanford, USA) was used to create the anatomical model to represent bone geometries and eleven muscle-tendon units: BicFemlh, Biceps femoris short-head (BicFemsh), GasLat, GasMed, RectFem, SemiMem, semi-tendinosus (SemiTen), TFL, VastInt, VastLat, and VastMed paths. The OpenSim muscle analysis tool was used to estimate the muscle-tendon lengths, adduction/abduction moment arms about the medial and lateral condyle contact points, and the flexion/extension moment arms from the lower limb kinematics during gait (Delp et al., 2007).

Muscle activation patterns were derived from the EMG data. The raw EMG signals were bandpass filtered (30-500 Hz), full wave rectified, low-pass filtered using a zero phase-lag Butterworth filter (4th order, $6 \mathrm{~Hz}$ cut-off frequency), and normalized by the maximal value of each muscle estimated on both maximal isometric contractions and gait trials. The muscle activation of SemiTend was assumed to be equal to that of SemiMem; BicFemlh and BicFemsh were assumed to be identical; and the muscle activation of VastInt was the average of that from VastLat and VastMed. The transformation from normalized EMG to muscle activation was obtained by including second-order dynamics, electromechanical delay and a non-linear relationship between EMG and muscle activation (Lloyd and Besier, 2003; Manal and Buchanan, 2003).

We estimated the individual muscle-tendon forces using a Hill-type muscle model (Zajac, 1989; Schutte 1992). Individual muscle forces were then multiplied by the muscle-tendon moment arms and summed to determine the net knee joint flexion/extension moments. Before applying the EMG-driven model to estimate muscle-tendon forces and joint contact forces, the model was first calibrated to each subject.

\subsection{Calibration and prediction process}

The EMG-driven model was calibrated to each subject by minimising three different cost functions that used the flexion/extension knee joint moments and joint contact forces from the instrumented implant. The model parameters (muscle activation parameters, strength coefficients, optimal fibre lengths, and tendon slack lengths) were calibrated using simulated annealing (Kirkpatrick et al., 1983) to minimise the following three calibration cost functions:

1. $\min \left[\Delta M^{K F E}\right]$ : by minimizing the difference between the knee joint flexion/ extension moments computed by inverse dynamics $\left(M_{I D}^{K F E}\right)$ and the EMG-driven model $\left(M_{M O D}^{K F E}\right)$, i.e.

$$
\min \left[\frac{1}{n} \sum_{i=1}^{n}\left\{\left(M_{I D}^{K F E}\left(t_{i}\right)-M_{M O D}^{K F E}\left(t_{i}\right)\right)^{2}\right\}\right] .
$$

2. $\min \left[\Delta M^{K F E}+\max F^{M C}+\max F^{L C}\right]$ : by minimizing i) the differences between the knee joint flexion/extension moments computed by inverse dynamics and the 
model, and ii) the maximum value of the modelled knee contact force at each condyle ( $F_{M O D}^{M C}$ and $F_{M O D}^{L C}$ ), i.e.

$$
\min \left[\frac{1}{n} \sum_{i=1}^{n}\left\{\left(M_{I D}^{K F E}\left(t_{i}\right)-M_{M O D}^{K F E}\left(t_{i}\right)\right)^{2}\right\}+w_{1}\left(\max F_{M O D}^{M C}\right)+w_{2}\left(\max F_{M O D}^{L C}\right)\right]
$$

where, $w_{i}$ are weight coefficients used for each parameter during calibration.

3. $\min \left[\Delta M^{K F E}+\Delta F^{M C}+\Delta F^{L C}\right]$ : by minimizing the differences i) between the knee joint flexion/extension moments computed by inverse dynamics and the model, and ii) between the knee contact force at each condyle computed by the model and measured by the instrumented implant $\left(F_{\text {invivo }}^{M C}\right.$ and $\left.F_{\text {invivo }}^{L C}\right)$, i.e.

$\left.\min \left[\frac{1}{n} \sum_{i=1}^{n}\left\{\left(M_{I D}^{K F E}\left(t_{i}\right)-M_{M O D}^{K F E}\left(t_{i}\right)\right)^{2}+w_{3}\left(F_{M O D}^{M C}\left(t_{i}\right)-F_{\text {invivo }}^{M C}\left(t_{i}\right)\right)^{2}+w_{4}\left(F_{M O D}^{L C}\left(t_{i}\right)-F_{\text {invivo }}^{L C}\left(t_{i}\right)\right)^{2}\right)\right\}\right]$

During the calibration process, the different weighting coefficients were also manually adjusted based on the error between the model estimates and the measurements (in vivo contact force and inverse dynamics results). The values were 1/6, 1/12, 1/200 and 1/400, respectively, for $w_{1}, w_{2}, w_{3}$ and $w_{4}$. Calibration was repeated for each model using each of the six overground gait trials with each of the three cost functions. However, for each cost function, the adjusted model parameter values that were used for further analyses were selected from the one gait trial that produced the lowest root mean squared error $\left(\mathrm{RMS}_{\text {error }}\right)$ between estimated and measured joint contact forces. These parameter values were then used to predict the muscle-tendon forces, knee-joint flexion/extension moments and kneejoint contact forces in the five additional walking trials not used for calibration.

\subsection{Estimation of knee-joint joint contact forces}

Joint contact forces were estimated using the point contact method, which has been described in detail elsewhere (Winby et al, 2009; Kumar et al, 2012). This method assumes the medial and lateral contact forces act through one single point for each compartment, separated by the inter-condyle distance. Briefly, the contact forces are determined using static equilibrium about the medial and lateral contact points in the tibial frontal plane. Using the medial contact force as an example calculation (Figure 2), the external adduction/ abduction moment, determined at the lateral contact point via inverse dynamics in OpenSim, was balanced by the muscle moments relative to the lateral contact point (i.e., the product of the muscle-tendon forces estimated by the EMG-driven approach and the muscle-tendon moment arms relative to the lateral contact point computed in OpenSim) and the unknown medial contact forces (Figure 2). The actions of the collateral ligaments were neglected. This process was repeated at every time step. The same approach was used to determine the lateral contact forces, where the muscle-tendon forces were the same, but the external adduction/abduction moments and muscle moment arms were determined about the medial condyle contact point.

\subsection{Data analysis}

Joint contact force predictions generated by the four different OpenSim models for the three different cost functions were analysed. Using these predicted results, the performance of each model and cost function combination was assessed using the RMS error between $M_{I D}^{K F E}$ and $M_{M O D}^{K F E}$, between experimental and models' medial contact forces, and between experimental and model lateral contact forces. Because results were available from only one 
subject, we calculated the $95 \%$ confidence intervals (CIs) for the RMS errors. If no overlap existed between the upper and lower bounds of the CIs, we assumed that differences existed in the prediction accuracies from the different conditions. We also compared the moments arms estimated by the different models to those published previously (Buford et al. 1997; Grood et al. 1984; Sobczak et al. 2013; Spoor et al. 1992) from selected major muscles for knee flexion angles from $0^{\circ}$ to $100^{\circ}$, and with hip and ankle joints each set at $0^{\circ}$ flexion. Finally, we examined how changes in moment arms across the different models affected muscle forces estimates in gait for selected major muscles.

\section{Results}

After the completion of the calibration process, the prediction accuracy of knee joint flexion/ extension moments was similar regardless of which model was used (Figure 3A). The $\mathrm{RMS}_{\text {errors }}$ ranged from 2.3 to 17.9 N.m, suggesting that the EMG driven algorithm was able to reproduce the net flexion/extension moments for any of the models and conditions.

A cost-function based only on the knee joint moment led to an overestimate of the medial contact forces irrespective of which geometric model and kinematics was used (Figures 34). When the cost function included constraints on the contact forces ( $\min \left[\Delta M^{K F E}+\max \right.$ $\left.F^{M C}+\max F^{L C}\right]$ or min $\left[\Delta M^{K F E}+\Delta F^{M C}+\Delta F^{L C}\right]$ ), the RMS errors were lower and the $95 \%$ CIs did not overlap the results obtained using only the knee joint moment (min $\left[\Delta M^{K F E}\right]$ ) for the medial and lateral contact forces. This finding indicates a better predictive accuracy of the measured medial and lateral contact forces (Figures 3-5) when quantities related to contact forces were included in the calibration cost function.

The accuracy of predicted medial contact forces was improved by using the subject-specific knee geometry. The subject-specific knee geometry models had medial contact forces estimates closer to in vivo measurements (Figure 3B). Interestingly, use of the subjectspecific kinematics did not improve estimates of medial and lateral contact forces.

The moment arms estimated using the SS-Geom \& SS-Kin and G-Geom \& G-Kin models were within the range of moment arms obtained from experiment (Buford et al. 1997; Grood et al. 1984; Sobczak et al. 2013; Spoor et al. 1992) for most of the muscles except for the quadriceps muscle group (Figure 6). The estimated moment arms (Figure 7B and D) and the muscle forces (Figure 7A and C) during gait were also different depending on the model used, although the shapes of the muscle force curves were similar (Figure 7A and $C$ ).

\section{Discussion}

The aim of this study was to investigate the influence of subject-specific knee joint geometry and kinematics on the estimation of tibiofemoral contact forces using an EMGdriven neuromusculoskeletal modelling approach. We hypothesized that subject-specific knee joint geometry and/or subject-specific knee joint kinematics would improve the accuracy of medial and lateral contact force predictions compared to using a generic model. The subject-specific knee joint geometric model improved the accuracy of estimated medial contact forces over the generic geometric model only when cost function terms involving knee contact forces were included during the calibration process. Therefore, our findings suggest that accurate joint geometry may be necessary to obtain close agreement between predicted and experimental medial knee joint contact forces. However, accurate geometry is not sufficient as muscle-tendon parameters also have to be adjusted to obtain the best possible agreement by minimizing peak of contact forces during the calibration process.

In this study, estimation of muscle forces was based on subject-specific muscle activation patterns derived from recorded EMG. Lin et al. (2010) solved the problem of muscle 
redundancy by using optimization to estimate knee joint contact forces. Whereas their method required an optimization process to estimate contact forces, the EMG-driven approach could be used to predict muscle and joint forces without optimization after completing an optimization-based calibration process, allowing the approach to be used for real-time applications. Furthermore, even though Lin and colleagues found close agreement with in vivo contact force measurements, they found different muscle force patterns depending on the optimization function employed. Use of EMG data constrained the solution space to reflect individual muscle activation patterns better. For this reason, in our study, the use of different cost functions during the EMG-driven model calibration process mainly affected the amplitudes of the muscle forces but not their shapes (Figure 6). Since Lin et al. (2010) modeled muscles as pure force generators without activation dynamics, and contraction dynamics, they achieved a better fit to in vivo measurements than in the present study. Nonetheless, we believe that using EMG data as model inputs provides better physiological estimation of muscle forces.

When the models were calibrated with cost function terms involving the joint contact forces, the generic geometric model produced higher muscle forces and overestimated medial contact forces compared to the subject-specific geometric model. This result is because the subject-specific geometry affected the moment arms and forces of several muscles (Figure 6) due changes in muscle-tendon paths and in the position of the knee joint centre. The larger extension moment arms led to lower muscle forces and thus lower contact forces as observed in the estimated medial contact force. These observations may explain the tendency of previous models to overestimate the tibiofemoral contact forces (Fregly et al., 2012).

In the subject-specific geometric model, each muscle-tendon path was adjusted manually, which may have introduced some errors. Advanced statistical analysis is needed to investigate the influence of variation introduced by manual adjustment. This manual step could be avoided by using magnetic resonance imaging and/or ultrasonography, although, using medical imaging does not guarantee accurate moment arm estimations due to the sensitivity of the methods to the positions of the muscles origins and insertions (Pal et al., 2007). Nevertheless, except for the quadriceps muscle groups, the muscle moment arms estimated by G-Geom \& G-Kin and SS-Geom \& SS-Kin models were within the range of those reported in experimental studies (Grood et al., 1984; Spoor et al. 1992; Buford et al. 1997; Sobczak et al. 2013). At least for the subject-specific model, the larger quadriceps moment arms could be explained by the knee prosthesis design. Specifically, the native patella has an added button that articulates with the femoral component in order to increase the quadriceps moment arms and reduce the quadriceps force (Figure 7C and D), thereby reducing joint contact forces (Browne et al., 2005). Nevertheless, further investigation is required to understand the influence of subject-specific bone geometry on the estimation of knee contact forces for healthy individuals as the knee prosthesis changes knee geometry.

Calibration of EMG-driven models is usually based on minimization of the difference between the joint moments computed by the model and by inverse dynamics (Lloyd and Besier 2003). In our study, using this approach led to large errors in estimates of knee joint contact forces. Not surprisingly, using in vivo knee contact force measurements during calibration considerably reduced contact force errors compared to the use of the knee joint moment alone. However, in vivo measurements of joint contact forces are rarely available. The calibration process that minimized the estimated peak contact forces, without use of in vivo measurements, improved the accuracy of contact force predictions compared to use of only the knee flexion/extension moment. Furthermore, this approach produced comparable accuracy to calibrations that employed the in vivo measurements. This improvement is directly related to the different values of model parameters adjusted during the calibration 
process. Among all model parameters, muscle strength coefficients were affected the most by the different calibration cost functions, though not by the choice of knee geometric model. Strength coefficients were used to scale maximal isometric force according to muscle group (knee flexor, knee extensor and knee flexor-ankle dorsiflexor) and ranged between 0.5 and 2.5. The additional cost functions that improved joint contact force estimates led to lower strength coefficients for all muscle groups compared to the use of only the knee joint moment during the calibration process. Lower strength coefficients could be explained by decreased muscle force with aging.

Despite improvements made by using subject-specific knee geometry, the EMG-driven approach did not predict the in vivo knee contact forces as closely as desired. Several reasons for this discrepancy are possible. We focused on subject-specific knee geometry and kinematics, but other muscle-tendon model parameter values can influence muscle force estimates. In the EMG-driven approach, even though muscle-tendon model parameters are calibrated, the solution space remains large due to the presence of muscle redundancy. Thus, calibrated parameter values may not represent the true subject-specific muscle-tendon properties. Li et al. (2009) have shown that the use of subject-specific muscle optimal fibre lengths and pennation angles improved the prediction of elbow movement for healthy individuals and individuals post-stroke. Furthermore, in the current study, the mechanical properties of tendon were generic whereas Gerus et al. (2012) have shown that use of subject-specific tendon force-strain relationships can influence the estimation of muscle force.

The current study suggests that for an EMG-driven modeling approach, the calibration cost function plays a large role in obtaining appropriate muscle-tendon model parameter values. We also used the measured contact force data to evaluate the best calibration, which is a potential limitation of our study. Further research is needed to determine the calibration cost functions that produce the best estimates of joint contact forces, without resorting to the measured contact forces. In the current and other studies (Kumar et al., 2012; Winby et al., 2009), the calibration cost functions used only the knee flexion/extension moment, whereas additional external measurements such as hip and ankle joint moments are also available. Some knee muscles are bi-articular and exert moments at the hip or ankle. The use of additional degrees of freedom in the calibration process would allow better constraining the EMG-dependent muscle force estimates while reducing the model's parameter solution space (Sartori et al., 2012). Furthermore, while EMG-driven approaches possess notable advantages over optimization methods, EMG measurements contain errors due to cross-talk from surrounding muscles, electrode placement, and impedance between the muscle and the electrode. EMG measurements are also limited to the use of surface electrodes, or to very specific sites using fine-wire EMG, and recording EMG from deep muscles is generally problematic. Combining calibration of an EMG-driven approach with subsequent optimization to adjust EMG-derived muscle activations (i.e. EMG-assisted optimization methods) may offer a solution to these issues for non-real-time applications. Another facet that could be improved is the contact model used to estimate knee contact forces, which in the current work is a simplified two-point contact representation. More complex contact models could be used, such as an elastic foundation model (Lin et al., 2010) or finite element modelling. The combination of EMG-assisted approaches to estimate muscle force with a validated contact model might improve the estimation of joint contact forces, which will be the focus of future work. Finally, since this study only used one subject, further work on the generalizability of the results needs to be performed. 


\section{Acknowledgments}

This work was supported by NIH grant-RO1-EB009351 and in part by the National Health and Medical Research Council in Australia [628850].

\section{References}

Ackland DC, Lin Y-C, Pandy MG. Sensitivity of model predictions of muscle function to changes in moment arms and muscle-tendon properties: A monte-carlo analysis. J Biomech. 2012; 45(8):14631471. [PubMed: 22507351]

Arnold EM, Ward SR, Lieber RL, Delp SL. A model of the lower limb for analysis of human movement. Annals of Biomedical Engineering. 2010; 38(2):269-279. [PubMed: 19957039]

Besier TF, Sturnieks DL, Alderson JA, Lloyd DG. Repeatability of gait data using a functional hip joint centre and a mean helical knee axis. J Biomech. 2003; 36(8):1159-1168. [PubMed: 12831742]

Buchanan TS, Lloyd DG, Manal K, Besier TF. Neuromusculoskeletal modeling: estimation of muscle forces and joint moments and movements from measurements of neural command. Journal of Applied Biomechanics. 2004; 20(4):367-395. [PubMed: 16467928]

Browne C, Hermida JC, Bergula A, Colwell CW Jr, D'Lima DD. Patellofemoral forces after total knee arthroplasty: effect of extensor moment arm. Knee. 2005; 12(2):81-8. [PubMed: 15749440]

Buford WL Jr, Ivey FM Jr, Malone JD, Patterson RM, Peare GL, Nguyen DK, Stewart AA. Muscle balance at the knee-moment arms for the normal knee and the ACL-minus knee. IEEE Trans Rehabil Eng. 1997; 5:367-379. [PubMed: 9422462]

Cheng C-K, Yao N-K, Liu H-C, Lee K-S. Influences of configuration changes of the patella on the knee extensor mechanism. Clin Biomech (Bristol, Avon). 1996; 11(2):116-120.

Crowninshield RD, Brand RA. A physiologically based criterion of muscle force prediction in locomotion. J Biomech. 1981; 14(11):793-801. [PubMed: 7334039]

Delp SL, Loan JP, Hoy MG, Zajac FE, Topp EL, Rosen JM. An interactive graphics-based model of the lower extremity to study orthopaedic surgical procedures. IEEE Transactions on Bio-Medical Engineering. 1990; 37(8):757-767. [PubMed: 2210784]

Delp SL, Anderson FC, Arnold AS, Loan P, Habib A, John CT, Guendelman E, Thelen DG. Opensim: open-source software to create and analyze dynamic simulations of movement. IEEE Transactions on Bio-Medical Engineering. 2007; 54(11):1940-1950. [PubMed: 18018689]

D'Lima DD, Townsend CP, Arms SW, Morris BA, Colwell CW Jr. An implantable telemetry device to measure intra-articular tibial forces. J Biomech. 2005; 38(2):299-304. [PubMed: 15598457]

Donnelly CJ, Lloyd DG, Elliott BC, Reinbolt JA. Optimizing whole body kinematics to minimize valgus knee loading during sidestepping: implications for ACL injury risk. J Biomech. 2012; 45(8):1491-1497. [PubMed: 22387123]

Duda GN, Brand D, Freitag S, Lierse W, Schneider E. Variability of femoral muscle attachments. J Biomech. 1996; 29(9):1185-1190. [PubMed: 8872275]

Foroughi N, Smith R, Vanwanseele B. The association of external knee adduction moment with biomechanical variables in osteoarthritis: a systematic review. Knee. 2009; 16(5):303-309. [PubMed: 19321348]

Fregly BJ, Besier TF, Lloyd DG, Delp SL, Banks SA, Pandy MG, D’Lima DD. Grand challenge competition to predict in vivo knee loads. J Orthop Res. 2012; 30(4):503-513. [PubMed: 22161745]

Gerus P, Rao G, Berton E. Subject-specific tendon-aponeurosis definition in Hill-type model predicts higher muscle forces in dynamic tasks. Plos One. 2012; 7(8):e44406. [PubMed: 22952973]

Grood ES, Suntay WJ, Noyes FR, Butler DL. Biomechanics of the knee-extension exercise. Effect of cutting the anterior cruciate ligament. J Bone Joint Surg Am. 1984; 66:725-734. [PubMed: 6725319]

Guilak F. Biomechanical factors in osteoarthritis. Best Pract Res Clin Rheumatol. 2011; 25(6):815823. [PubMed: 22265263]

Hamner SR, Seth A, Delp SL. Muscle contributions to propulsion and support during running. Journal of Biomechanics. 2010; 43(14):2709-2716. [PubMed: 20691972] 
Heiden TL, Lloyd DG, Ackland TR. Knee joint kinematics, kinetics and muscle co-contraction in knee osteoarthritis patient gait. Clin Biomech. 2009; 24(10):833-841.

Hurwitz DE, Sumner DR, Block JA. Bone density, dynamic joint loading and joint degeneration. a review. Cells Tissues Organs. 2001; 169(3):201-209. [PubMed: 11455115]

Kirkpatrick S, Gelatt CD Jr, Vecchi MP. Optimization by simulated annealing. Science. 1983; 220(4598):671-680. [PubMed: 17813860]

Kumar D, Manal KT, Rudolph KS. EMG-driven modeling approach to muscle force and joint load estimations: case study in knee osteoarthritis. J Orthop Res. 2012; 30(3):377-383. [PubMed: 21901754]

Li L, Tong KY, Hu XL, Hung LK, Koo TKK. Incorporating ultrasound-measured musculotendon parameters to subject-specific EMG-driven model to simulate voluntary elbow flexion for persons after stroke. Clinical Biomechanics. 2009; 24:101-109. [PubMed: 19012998]

Lin Y-C, Walter JP, Banks SA, Pandy MG, Fregly BJ. Simultaneous prediction of muscle and contact forces in the knee during gait. J Biomech. 2010; 43(5):945-952. [PubMed: 19962703]

Lloyd DG, Besier TF. An EMG-driven musculoskeletal model to estimate muscle forces and knee joint moments in vivo. Journal of Biomechanics. 2003; 36(6):765-776. [PubMed: 12742444]

Manal K, Buchanan TS. A one-parameter neural activation to muscle activation model: estimating isometric joint moments from electromyograms. Journal of Biomechanics. 2003; 36(8):11971202. [PubMed: 12831746]

Meyer AJ, D’Lima DD, Besier TF, Lloyd DG, Colwell CW Jr, Fregly BJ. Are external knee load and EMG measures accurate indicators of internal knee contact forces during gait? Journal of Orthopaedic Research. 2012 In press.

Pal S, Langenderfer JE, Stowe JQ, Laz PJ, Petrella AJ, Rullkoetter PJ. Probabilistic modeling of knee muscle moment arms: effects of methods, origin-insertion, and kinematic variability. Ann Biomed Eng. 2007; 35(9):1632-1642. [PubMed: 17546504]

Roemhildt ML, Beynnon BD, Gardner-Morse M, Badger G, Grant C. Changes induced by chronic in vivo load alteration in the tibiofemoral joint of mature rabbits. J Orthop Res. 2012

Sartori M, Reggiani M, Pagello E, Lloyd DG. EMG-driven forward-dynamic estimation of muscle force and joint moment about multiple degrees of freedom in the human lower extremity. Plos One. 2012; 7(12):e52618. [PubMed: 23300725]

Scheys L, Desloovere K, Suetens P, Jonkers I. Level of subject-specific detail in musculoskeletal models affects hip moment arm length calculation during gait in pediatric subjects with increased femoral anteversion. J Biomech. 2011; 44(7):1346-1353. [PubMed: 21295307]

Schipplein OD, Andriacchi TP. Interaction between active and passive knee stabilizers during level walking. J Orthop Res. 1991; 9(1):113-119. [PubMed: 1984041]

Schutte, LM. PhD Thesis. Stanford University; 1992. Using musculoskeletal models to explore strategies for improving performance in electrical stimulation induced leg cycle ergometry.

Sobczak S, Dugailly PM, Feipel V, Baillon B, Rooze M, Salvia P, Van Sint Jan S. In vitro biomechanical study of femoral torsion disorders: Effect on moment arms of thigh muscles. Clinical Biomechanics. 2013; 28(2):187-192. [PubMed: 23337767]

Solomon L. Patterns of osteoarthritis of the hip. J Bone Joint Surg Br. 1976; 58(2):176-183. [PubMed: 932079]

Spoor CW, van Leeuwen JL. Knee muscle moment arms from MRI and from tendon travel. J Biomech. 1992; 25:201-206. [PubMed: 1733995]

Tsai L-C, Colletti PM, Powers CM. Magnetic resonance imaging-measured muscle parameters improved knee moment prediction of an EMG-driven model. Med Sci Sports Exerc. 2012; 44(2): 305-312. [PubMed: 21775905]

Winby CR, Lloyd DG, Besier TF, Kirk TB. Muscle and external load contribution to knee joint contact loads during normal gait. J Biomech. 2009; 42(14):2294-2300. [PubMed: 19647257]

Zajac FE. Muscle and tendon: properties, models, scaling, and application to biomechanics and motor control. Critical Reviews in Biomedical Engineering. 1989; 17(4):359-411. [PubMed: 2676342]

Zeni JA, Rudolph K, Higginson JS. Alterations in quadriceps and hamstrings coordination in persons with medial compartment knee osteoarthritis. J Electromyogr Kinesiol. 2010; 20(1):148-154. [PubMed: 19223203] 
Zhao D, Banks SA, D'Lima DD, Colwell CW Jr, Fregly BJ. In vivo medial and lateral tibial loads during dynamic and high flexion activities. J Orthop Res. 2007; 25(5):593-602. [PubMed: 17290383] 


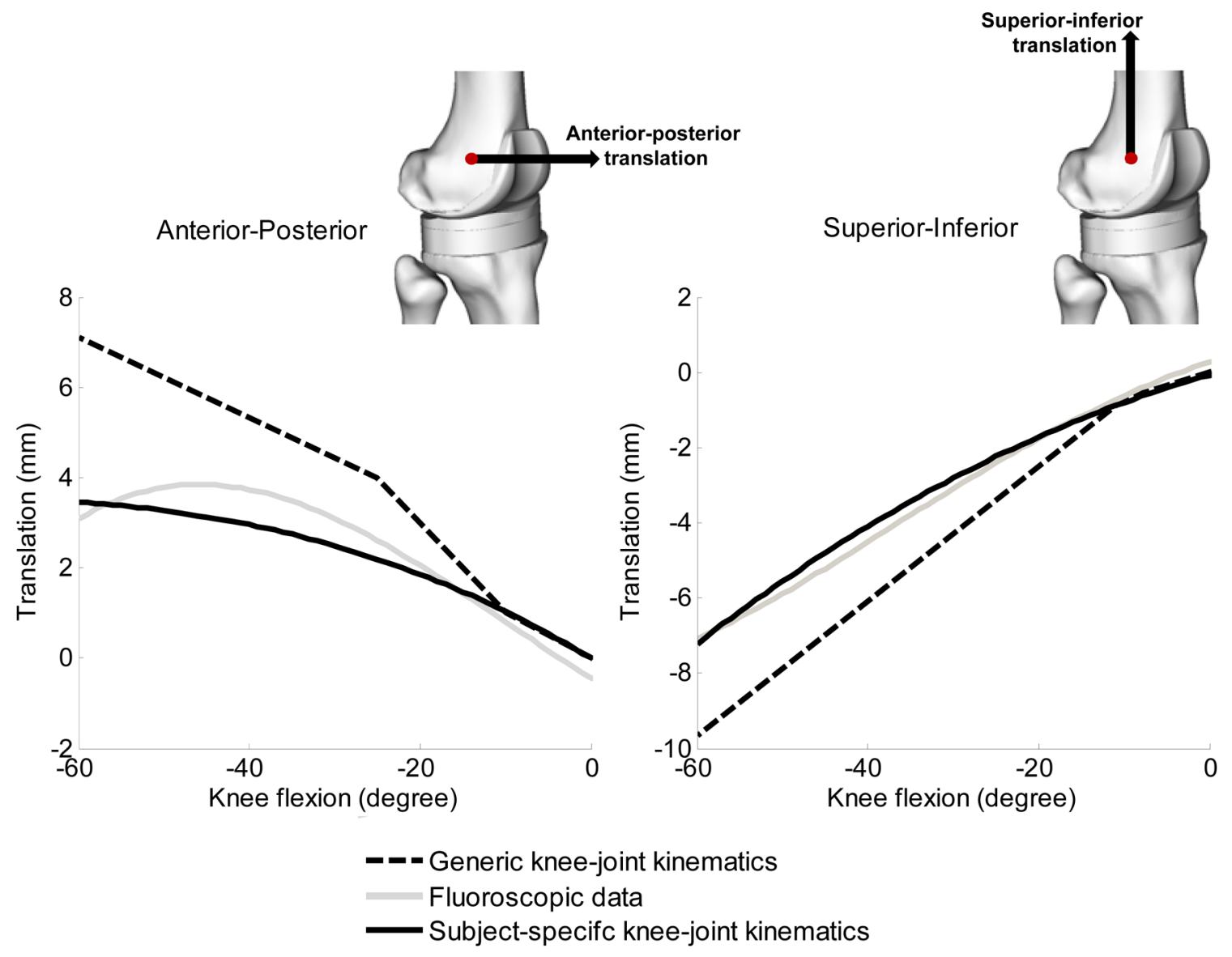

Figure 1.

Anterior-posterior and superior-inferior translation of the tibia relative to the femur defined in OpenSim for the generic and subject-specific knee joint kinematic model using fluoroscopic data. The reference $(0,0)$ is the initial position of the knee joint center, and the translation is the displacement of the knee joint center relative to its initial position. 


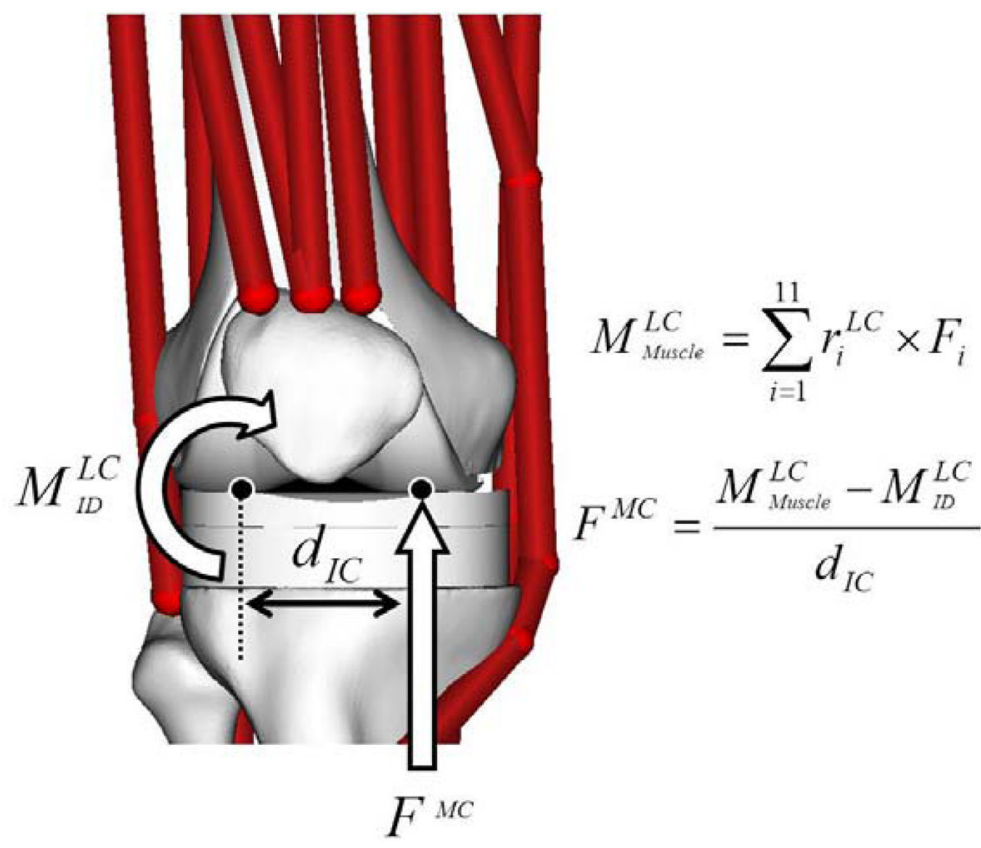

Figure 2.

As an example, a schematic of point contact model used to estimate the medial contact force. The external adduction/abduction moment about the lateral condyle $\left(M_{I D}^{L C}\right.$ ) estimated from OpenSim must be balanced by the moment produced by the muscles $\left(M_{M u s c l e}^{L C}\right)$ and an unknown contact force $\left(F^{M C}\right)$ acting at distance $\left(d_{I C}\right)$ equal to $40 \mathrm{~mm} . r_{i}^{L C}$ represents the muscle moment arms relative to the lateral contact point estimated by the OpenSim model and $F_{i}$ represents the muscle-tendon forces estimated by the EMG-driven approach. The same approach was used to estimate the lateral contact force, except moment arms and moments were determined about the medial condyle contact point. 

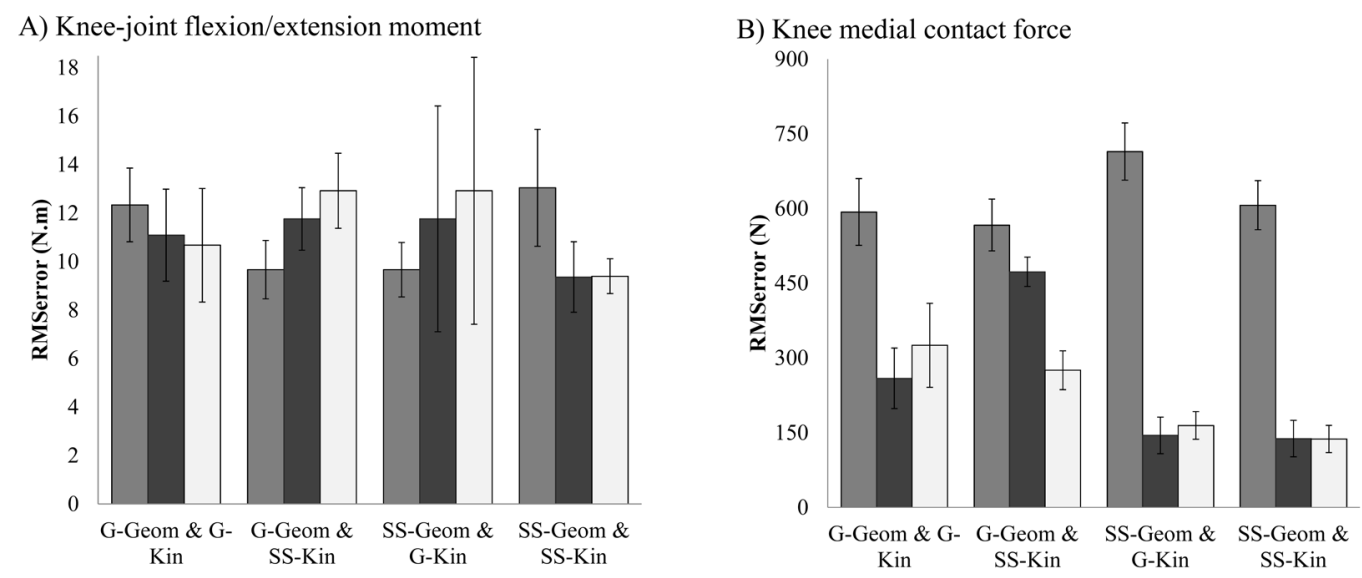

C) Knee lateral contact force
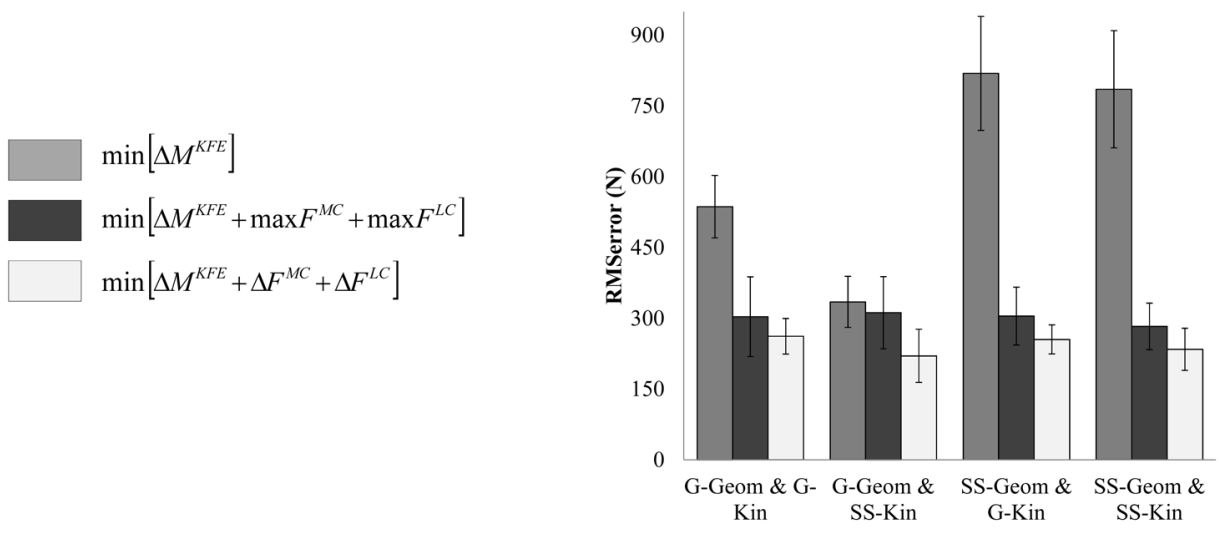

Figure 3.

$\mathrm{RMS}_{\text {errors }}$ estimated on (A) the knee joint flexion/extension moments, (B) the medial tibiofemoral contact forces, and (C) the lateral tibiofemoral contact forces in the walking trials using the different cost functions and knee joint models. The $\mathrm{RMS}_{\text {errors }}$ are the mean and the error-bars represents $95 \%$ confidence interval for the five prediction trials. 
(A)

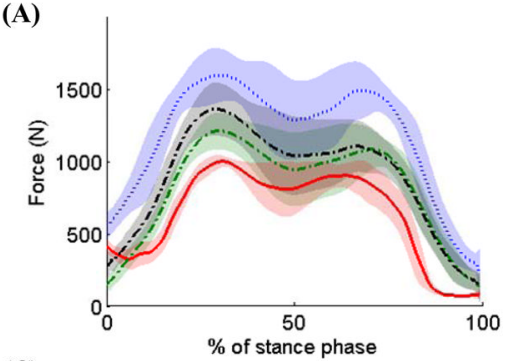

(C)

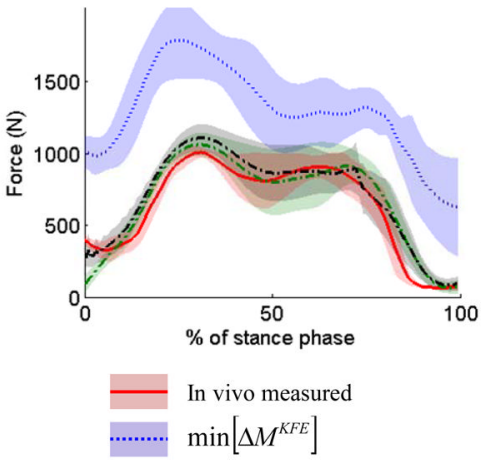

(B)

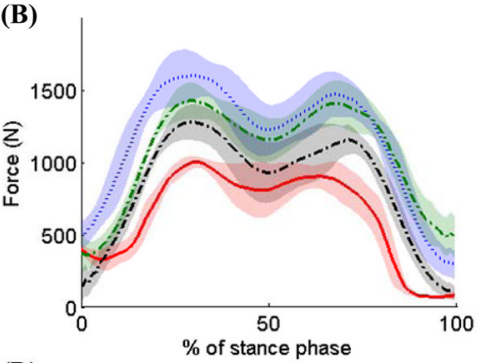

(D)

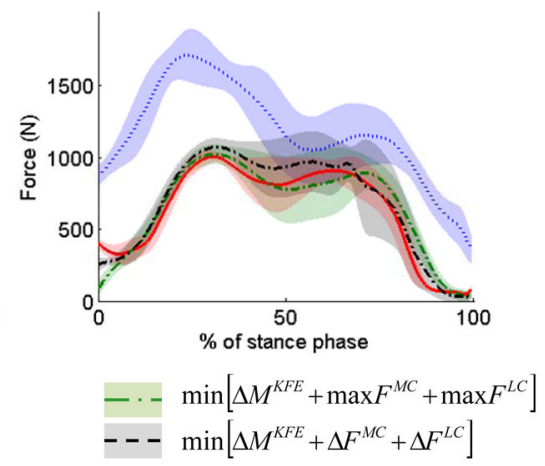

Figure 4.

Medial contact forces predicted from the (A) G-Geom \& G-Kin, (B) G-Geom \& SS-Kin, (C) SS-Geom \& G-Kin and (D) SS-Geom \& SS-Kin models and directly measured with the instrumented knee prosthesis (in vivo measured). Mean and standard deviations from five prediction trials are shown (shade regions). 
(A)

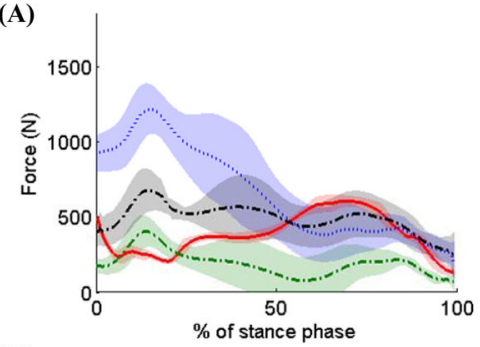

(C)

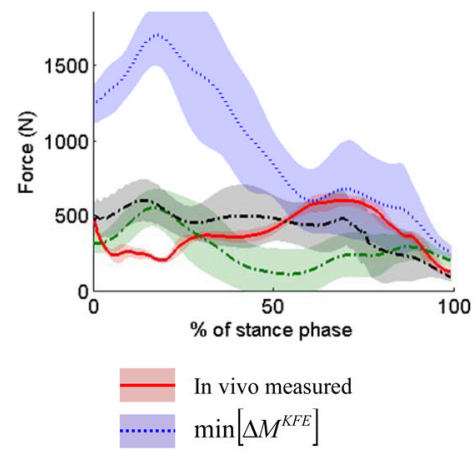

(B)

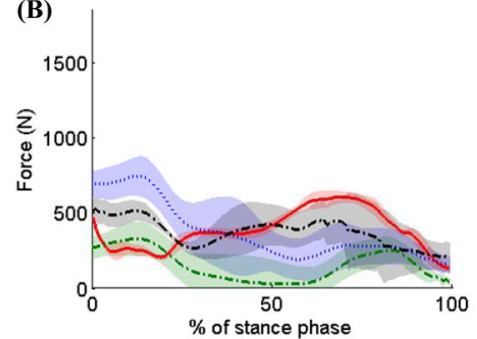

(D)

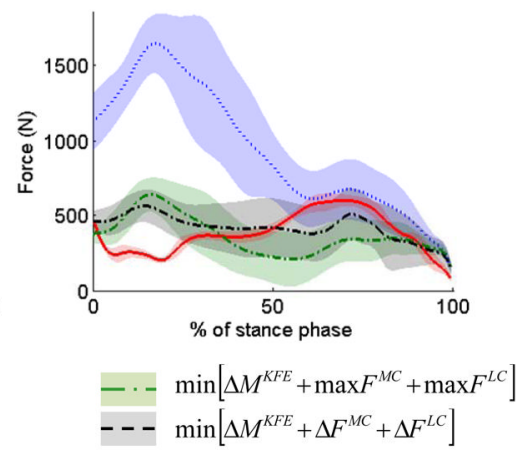

Figure 5.

Lateral contact forces predicted from the (A) G-Geom \& G-Kin, (B) G-Geom \& SS-Kin, (C) SS-Geom \& G-Kin and (D) SS-Geom \& SS-Kin models and directly measured with the instrumented knee prosthesis (in vivo measured). Mean and standard deviations from five prediction trials are shown (shade regions). 
(A)

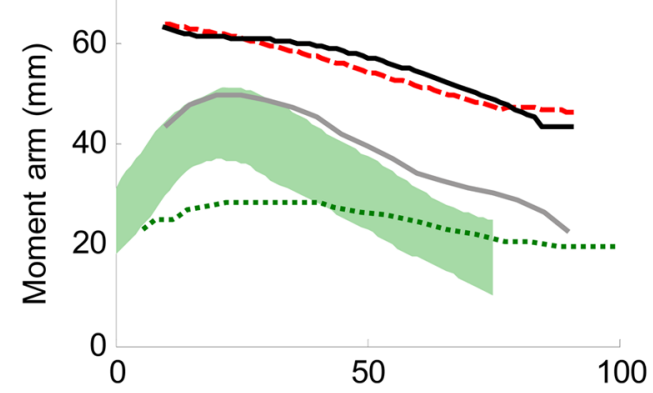

(C)

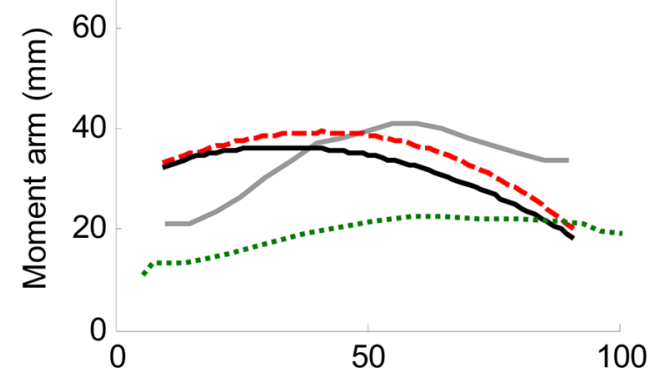

(E)

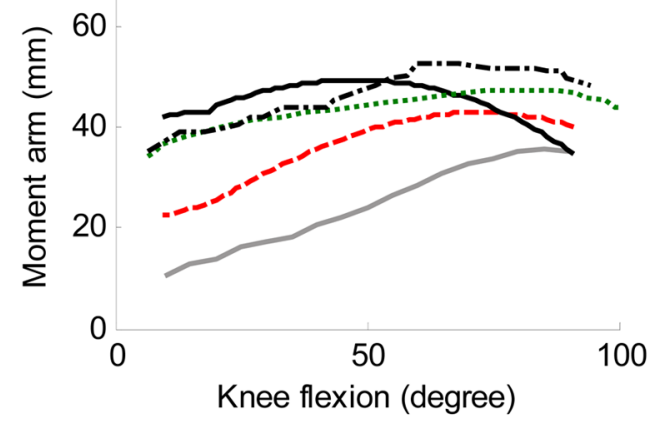

(B)

TFL

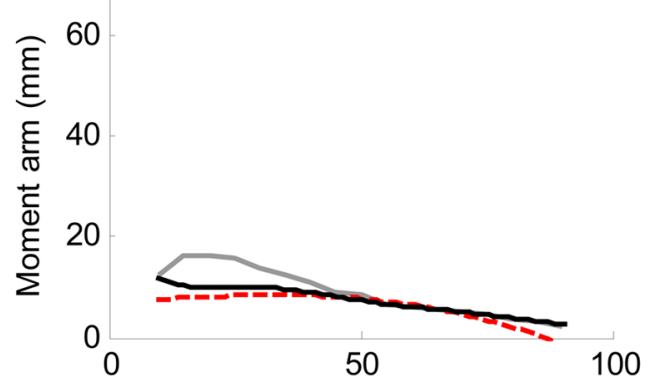

(D)

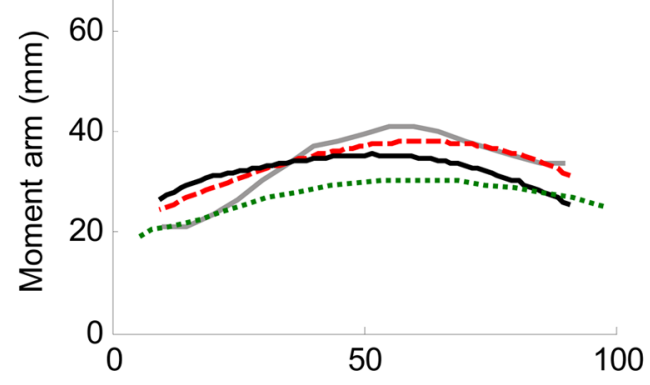

(F)

SemiMem

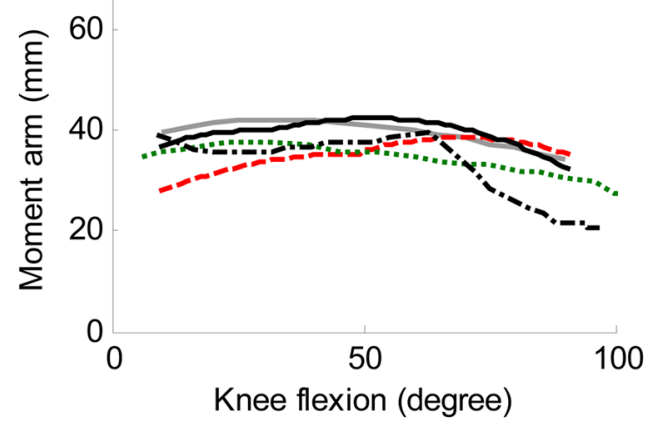

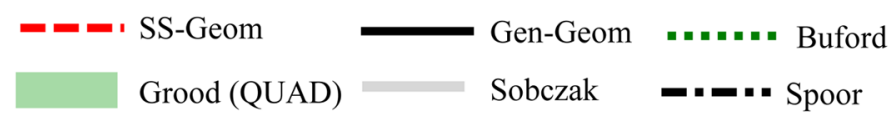

Figure 6.

Moment arms of selected major muscles estimated by the G-Geom \& G-Kin (dashed), the SS-Geom \& SS-Kin models (solid), and those measured experimentally (Buford et al., 1997; Grood et al., 1984; Sobczak et al. 2013; Spoor et al. 1992) for knee flexion angles from $0^{\circ}$ to $100^{\circ}$, and with hip and ankle joints each set at $0^{\circ}$ flexion. 

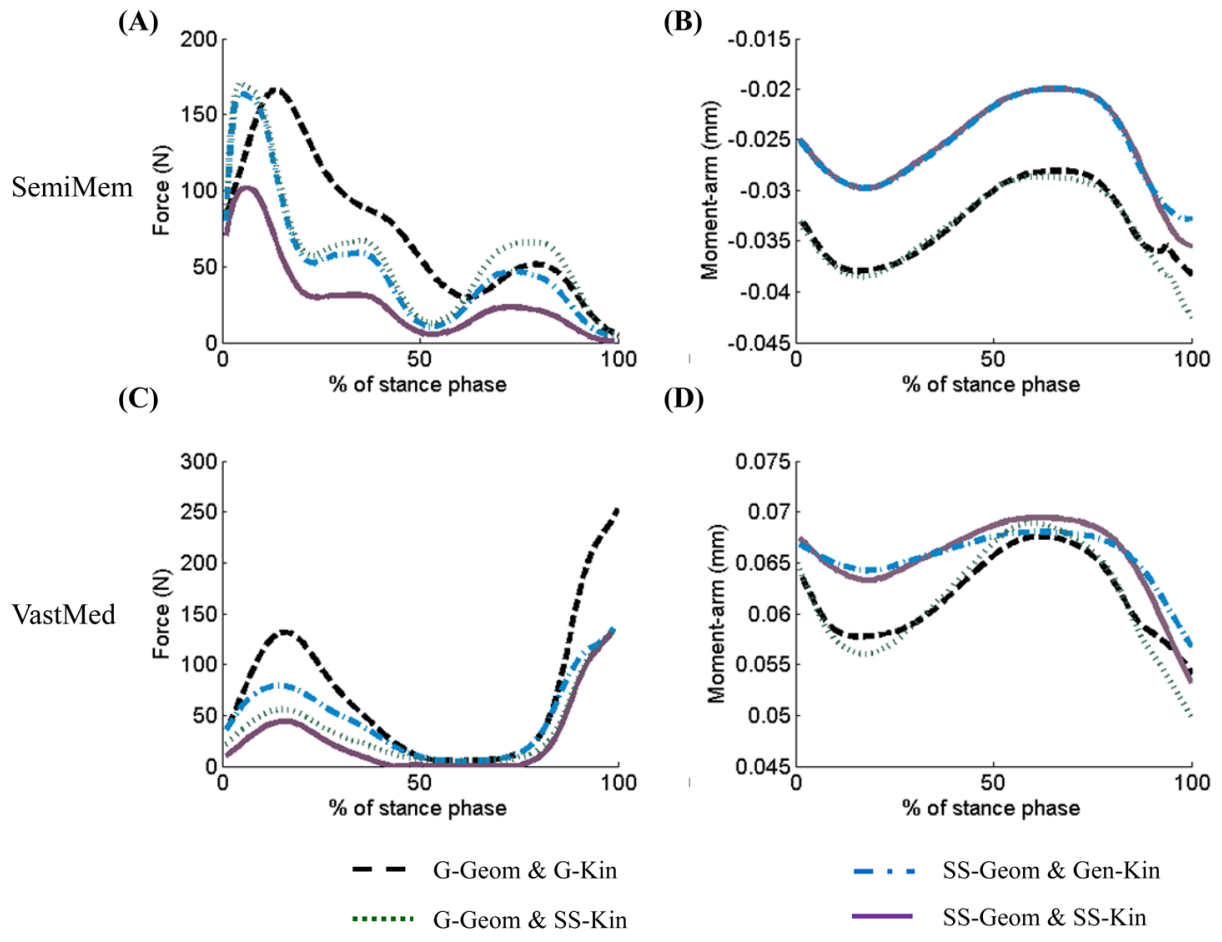

Figure 7.

Example of the muscle forces from the SemiMem (A) and VastMed (C), and the corresponding muscle-tendon moment arms from the SemiMem (B) and VastMed (D) obtained from the calibration gait trials for each EMG-driven model using the different knee joint geometry and kinematic models employing the $\min \left[\Delta M^{K F E}+\Delta F^{M C}+\Delta F^{L C}\right] \operatorname{cost}$ function. 